\title{
NEURAL NETWORKS FOR THE SIMULATION OF MICROCLIMATIC PARAMETERS IN DAIRY HOUSES
}

\author{
Alessandro D’Emilio, Rosalia Mazzarella, Simona M.C. Porto
}

\section{Introduction}

Environmental conditions inside livestock buildings considerably affect health and productivity of the housed animals [Bray 1997; Frazzi 2003].

Among the passive systems for environmental control of dairy houses, natural ventilation takes on particular importance as it represents the most efficient way to modify the thermo-hygrometric condition of the air and to reduce the concentration of noxious gases and dust.

According to the climate conditions, a correct design of a natural ventilation system should take into account the orientation and the height of the building, the geometry of the roof, the form, the size and the position of the openings for air exchanges [Cascone 1991; Holmes 1994; Stowell 2003; Liberati 2004; Zappavigna 2005].

The theoretical approach generally adopted to determine the natural ventilation flow for a livestock building consists of setting up a simplified energy balance of sensible heat flows, based on the hypothesis that all the heat or the water vapour produced by the animals is removed from the environment by ventilation. This approach however has some drawbacks, particularly when applied to the hottest hours of the days, because it does not take into account the contribution of the sun radiation, the energy quota exchanged in the form of latent heat and the wind contribution on the ventilation inside the building.

In the last years, Computational Fluid Dynamics (CFD) analysis was used for studying the ventilation conditions inside livestock buildings [Bjerg 2004; Wagenberg 2004; Cascone 2006]. Nevertheless, this kind of analysis, based on the numerical integration of

Paper received 10.07.2008; accepted 20.03.2009

ALESSANDRo D'Emilio, associate professor, ademilio@unict.it, RosAlia Mazzarella Phd, Simona M.C. Porto, Phd, University of Catania, Department of Agricultural Engineering, via S. Sofia 100-95123 Catania (Italy)

The authors' contribution is equal in every way. the differential equations describing the physical phenomenon of air motion, requires a very accurate modelling of both the building and the surroundings to provide reliable results.

Artificial neural network (ANN) models represent an alternative method in the study of several physical problems and are widely accepted as they offer the way to tackle complex and ill-defined systems.

Artificial neural networks are computational adaptive systems that change their structure in consequence of external inputs through a learning process. Among the main features, ANNs do not need detailed information about the system to be simulated and are able to handle noisy and incomplete data and to solve non linear problems. On the basis of these properties, several studies were carried out concerning the use of neural network models in practical applications such as designing control systems, solving recognition and classification problems, simulating complex physical problems [Towmey 1995; Kalogirou 2001; Cascone 2007].

The aim of the present paper is to study natural ventilation in a dairy house by means of a parametric analysis relating wind speed and direction to the air flows through the ridge vent of the building.

This analysis was carried out by means of a neural network model which capability in modelling and simulating some climatic parameters (air speed and direction through the ridge vent, through the sidewall openings and near the rack, air temperature and humidity) inside a dairy house has been validated.

\section{Materials and methods}

\subsection{Field trial}

A trial was carried out during summer 2005 in a cubicle dairy house (Figure 1) sited on the Hyblean Plateau (Sicily, Italy) at the altitude of $670 \mathrm{~m}$ above sea level. The barn is an open side building $24.55 \mathrm{~m}$ wide and $40.30 \mathrm{~m}$ long with the longitudinal axis lying on N-S direction. The building has a gable roof with unequal pitches. The pitch facing East is $7.45 \mathrm{~m}$ high 
at the ridge and $3.45 \mathrm{~m}$ high at the eave, the pitch facing West is $8.65 \mathrm{~m}$ high at the ridge and $5.70 \mathrm{~m}$ high at the eave. The continuous ridge vent facing East was equipped with a motorized adjustable shutter.

The building has a composite steel-concrete structure, with concrete frames located along the perimeter and a steel frame sited along the projection of the ridge. Along the cross-wise direction the roof has a steel-frame main structure bearing corrugated fibrecement sheets over wooden boards and steel purlins.

The East front of the building is completely open on a paved paddock, whereas the West front is partially closed by a wall $2.60 \mathrm{~m}$ high. Other doors and windows are symmetrically placed on the external walls facing North and South.

The rack divides the barn in two functional areas. The East side houses the rest zone, with 54 cubicles on two rows facing each other, whereas the West side of the building is used as feeding alley for a width of about $4.75 \mathrm{~m}$ and as a storage area for feed for a width of about $6.55 \mathrm{~m}$.

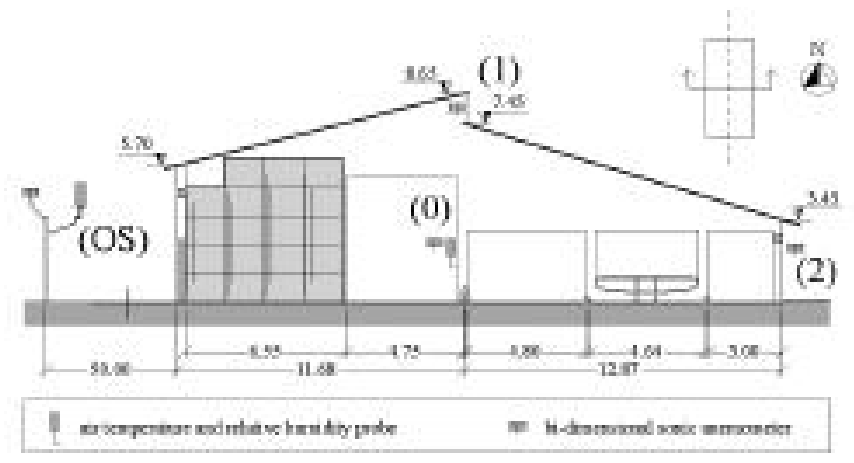

Fig. 1 - Cross section A-A of the dairy house where the trial was carried out with the arrangement of the sensors used in the study.

During the trial, several climatic and microclimatic parameters were measured outside and inside the barn respectively. For the purpose of this study only a subset of the monitored variables were used. In detail, air temperature and humidity, wind speed and direction were considered among the parameters measured outdoor (OS) (fig. 1). Inside the barn the measures of the following parameters monitored in the middle crosssection of the building were used: air temperature and humidity in the feed passage as well as air speed and direction near the rack (0), through the ridge vent (1) and through the opened East side (2). Air speed and direction were monitored by bi-dimensional sonic anemometers placed to measure the component of the air velocity on the horizontal plane.

The sensors inside the barn were placed at the height of $2.00 \mathrm{~m}$ above the floor, except for the anemometer placed at about half height of the ridge vent.

Air temperature and humidity were measured by means of a combined sensor provided with a platinum thermo-resistance and an hygrometer with radiation shield; for air temperature measurement the range is $40 \pm 60{ }^{\circ} \mathrm{C}$ and the accuracy is $\pm 0.2^{\circ} \mathrm{C}$ (at $20^{\circ} \mathrm{C}$ ), for air relative humidity measurement the dependence on temperature is $\pm 0.04 \%$ per ${ }^{\circ} \mathrm{C}$ and the accuracy is $\pm 2 \%$ (at $20^{\circ} \mathrm{C}$ ). Air speed and direction were measured by means of a bidimensional ultrasonic anemometers with measurement range of $0.01 \pm 60 \mathrm{~ms}^{-1}$, accuracy of $\pm 2 \%$ at $12 \mathrm{~ms}^{-1}$ for speed and of $\pm 3^{\circ}$ at $12 \mathrm{~ms}^{-1}$ for direction.

All the sensors were connected to data-loggers that read the measurements every 15 seconds and recorded the corresponding average values every 15 minutes.

\subsection{Neural network modelling}

An artificial neural network (ANN) is a computational system that emulates the behaviour of the biological nervous system. It is composed by basic computation units, which can be called variously "neurons", "Processing Elements" (PE) or "nodes", linked by synaptic connections each associated with a numerical value called "weight". An ANN acquires the knowledge by a learning process aimed to fix the value of each weight. Once ended the learning process, the network is ready to be used.

The way in which the neurons are grouped and linked characterizes the layout of the network. The neurons are generally arranged in parallel to form layers that can be classified as input layers, hidden layers and output layers. The input layer receives data from outside the network and has a number of neurons equal to the number of the input variables. The hidden layers contain neurons which signals keep inside the network. The output layer gives the result of the calculation and has a number of neurons equal to the number of the output variables.

In detail, a neuron receives data from all the input connections taking the weights into account and, through an activation function, computes the output value which is propagated to other neurons of the network. In the simplest case, when a pattern $p$ is presented to the network as a set of input data, the total input to the $k$ neuron is given by the weighted sum of the outputs from the neurons connected to $k$ plus an offset term (also called bias) [Kröse 1996; Cammarata 1997]:

$$
s_{k}^{p}=\sum_{j} w_{k} y_{j}^{j}+\theta_{j}^{p}
$$

where:

$s_{i}^{p}$ is the total input to the $k$ neuron;

$w_{\mathrm{s}}$ is the weight of the connection between the neurons $j$ and $k$;

$y_{j}^{\prime}$ is the output from the neuron $j$;

$\theta_{i}$ is the bias of the neuron $k$.

The new value of the output $y_{k}$, also called "activation value", is generally calculated applying the activation function $F_{k}$ to the total input:

$$
y_{j}^{\prime}=F_{j}\left(s_{k}^{b}\right)
$$

that is often a non-decreasing function of the total in- 
put (i.e.: threshold function, linear or semi-linear function, sigmoid function, hyperbolic tangent function, etc.).

The peculiarity of a neural network consists of its ability to learn. Neural nets are not programmed to solve a specific problem but they can be trained to solve it. The training is an iterative process that induces the changing of the connection weights through the execution of an appropriate algorithm based on different learning rules.

Two different paradigms of learning are available for modelling a neural network: unsupervised and supervised learning.

In this study the supervised learning was used being the more suitable for simulating physical problems. In supervised learning the network is trained by the presentation of examples of inputs together with the corresponding outputs. The network learns to infer the relationship between the pairs of input-output examples. In this case, the algorithms commonly used are based on the achievement of the minimum error between desired values and computed output in the shortest time.

One of the more used supervised algorithms is the Back-Propagation [Rumelhart 1986] that works in two phases. In the first phase an input pattern $p$ is presented to the network together with the corresponding desired output values. The input data are propagated forward through the network to obtain the calculated output. For each training iteration, the comparison between calculated and desired values gives the error signal for each output neuron. Consequently, the mean square error of the network is given by:

$$
E^{p}-\frac{1}{2} \sum_{s=1}^{y}\left(d_{o}^{p}-y_{*}^{p}\right)^{2}
$$

where

$N_{o}$ is the number of the output neurons;

$d_{0}$ is the desired output for the output neuron $o$;

$y^{F}$ is the current value of activation of the output neuron $o$.

The second phase involves a backward pass through the network during which the error signal is propagated to each neuron in the network and appropriate weight changes are calculated.

This process induces the change in the weight proportional to the negative of the derivative of $E^{p}$ :

$$
\Delta w_{\mu}=-\gamma \frac{\partial E^{P}}{\partial w_{\mu}}
$$

where $\gamma$ is a positive constant of proportionality called "learning rate", or "step size", that determines the amount of the weight adjustments during each training iteration.

Using the chain rule, the (4) can be written as:

$$
\Delta w_{i \mu}=-\gamma \frac{\partial E^{p}}{\partial s_{i}^{\prime}} \frac{\partial s_{i}^{f}}{\partial w_{j}}
$$

The first factor in the second term of the (5) is set:

$$
\delta_{i}^{p}=-\frac{\partial E^{p}}{\partial s_{i}^{p}}
$$

and stands for the change in error as a function of the net input to the neuron.

Applying (1), the second factor of the equation results:

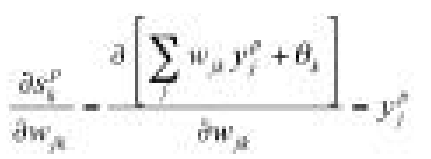

Substituting (6) and (7) in (5), it results:

$$
\Delta w_{A}=\gamma \delta_{i}^{\prime} y_{j}^{p}
$$

This relation is known as "generalised delta rule" and allows to change the weights according to a gradient descendent on the error surface.

In equation (8) the term $\delta_{;}^{F}$ can be calculated by a simple recursive computation implemented by propagating error signals backward through the network. In detail, using the chain rule, equation (6) can be rewritten as the product of two factors, one reflecting the change in error as a function of the neuron output and the other reflecting the change in the output as a function of changes in the total input:

$$
\delta_{j}^{\prime}=-\frac{\partial E^{y}}{\partial y_{k}^{v}} \frac{\partial y_{k}^{z}}{\partial s_{j}^{f}}
$$

Following the relation (2), under the assumption that the activation function $F_{k}$ is differentiable respect to the total input , the last factor of (9) becomes:

$$
\frac{\partial y_{f}^{\prime}}{\partial s_{f}^{\prime}}=F^{\prime}\left(x_{i}^{\prime}\right)
$$

To compute the first factor of equation (9), two cases have to be considered: first, $k=o$, that is the neuron $k$ is an output neuron, and secondly $k=h$, that is $k$ is a hidden neuron.

When $k=o$ from the definition (3) of $E^{p}$ follows that:

$$
\frac{\partial E^{p}}{\partial y_{0}^{p}}=-\left(d_{s}^{p}-y_{s}^{p}\right)
$$

Substituting this one and (10) in equation (9), it results:

$$
\delta_{s}^{z}=\left(d_{s}^{p}-y_{0}^{z}\right) F_{*}^{\prime}\left(s_{0}^{p}\right)
$$

This relation allows to calculate $\delta_{o}^{p}$ for any PE belonging to the output layer.

When $k=h$, it is possible to write the mean square error of the network as a function of the total inputs to the neurons of the output layer coming from the hidden layer:

$$
E^{r}=E^{p}\left(s_{1}^{r}, s_{1}^{p}, \ldots \ldots, s_{p}^{p}\right)
$$

Using the chain rule, the first term of equation (9) can be written:

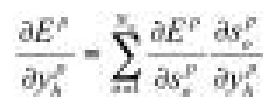

Substituting the (1) and simplifying: 


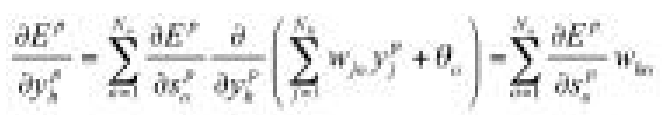

That for the (6) becomes:

$$
\frac{\partial E^{p}}{\partial y_{k}^{p}}=-\sum_{i=1}^{N} \delta_{s}^{p} w_{i}
$$

Substituting (10) and (16) in equation (9) yields:

$$
\delta_{h}^{z}=F^{\prime}\left(s_{h}^{p}\right) \sum_{i=1}^{N} \delta_{s}^{p} w_{i n}
$$

Equations (12) and (17) give a recursive procedure for computing the $\delta_{h}^{p}$ for all the neurons in the network, which are then used to compute the weight changes according to equation (8).

This procedure constitutes the "generalised delta rule" for a feed-forward network of non-linear neurons.

Each complete cycle of presentation of the training set to the network is defined "epoch". Generally a great number of epochs (thousands) is necessary before the network is able to produce a satisfactory output. The choice of a large value for the learning rate $\gamma$, that is generally fixed in the range between 0.05 and 0.75 , allows to speed up the convergence but, on the other hand, it could increase the risk of oscillations. A method for reducing this risk is to change the weights also in dependence of the previous weight change by adding a term $m$ called "momentum factor" [Hagivara 1992]:

$$
\Delta w_{g}=\gamma \delta_{k}^{f} y_{j}^{r}+m\left(w_{j}^{r}-w_{j}^{\prime-1}\right)
$$

The momentum factor (that is a positive number not larger than 0.9) gives the system a certain amount of inertia, as it generally smoothes the weight changes. But, at the same time, when the weight changes are all in the same direction, the momentum amplifies the learning rate, causing a faster convergence.

The criteria used to stop the learning is an important issue for obtaining a neural network able to both closely simulate the train data and generalize the results to unknown input data.

According to the simplest methods, the end of the training happens either after a pre-established number of epochs, or after the mean square error of the network is within the desired tolerance. Nevertheless neither approach offers any control on the generalization ability of the network. To overcome this limitation, various alternatives were proposed and among them the cross-validation is one of the most used. This procedure consists in checking the network performances at each iteration on a set of data not used for training. The training stops when the performances (generally the mean square error) on the crossing data do not improve further after an established number of iterations.

As the training of a net is heavily influenced by the initial values of the weights, several training cycles are generally carried out starting with different sets of randomly chosen values. The cycle with the best performance in terms of approximation and generalization is chosen.

At the end of the learning the values of all the weights are saved and kept constant. It is possible to verify the ability of the network in generalizing the results using a new set of data (testing set). If the output obtained with the input patterns of the testing set is not satisfactory, then it is necessary to change some parameters of the network, otherwise the network is ready to be used.

\subsection{Characteristics of the neural network used in this study}

In modelling physical processes, one of the most used neural networks is the Multi Layers Perceptron (MLP) in which any neuron of a "hidden" layer receives input from all the neurons of the previous layer and sends its output to the neurons of the following layer. No connections are present between neurons of the same layer or between neurons of non adjacent layers. As the signals propagate only from the input to the output, these types of networks are called "feed-forward".

The network architecture used in this study is a generalized feed-forward with two hidden layers. The difference from an ordinary MLP is that connections can jump over one or more layers. This kind of network was chosen as it provided the best results after several attempts.

The input layer has 4 neurons corresponding to the following parameters: outside air temperature and humidity and wind speed components in a Cartesian coordinate system on the horizontal plane. Specifically, the air speed components were calculated transforming the measures of wind speed and direction in a coordinate system with the $\mathrm{x}$-axis parallel to the cross section plan of the building and East-oriented and the $\mathrm{y}$-axis parallel to the longitudinal axis of the building and North-oriented. The output layer has 8 neurons corresponding to the following parameters: air speed components, expressed in the same Cartesian coordinate system, in the middle cross-section of the dairy house near the rack (0), through the ridge vent (1) and through the opened East side (2), and inside air temperature and humidity near the rack. The first and the second hidden layers contain 40 and 50 neurons respectively. The choice of the input variables is founded on their physical relation with the parameters to be simulated, the easiness of the measurement and the wide availability at the meteorological agencies.

The activation function for the hidden layers was the hyperbolic tangent:

$$
F_{i}\left(s_{k}^{p}\right)=\tanh \left(s_{i}^{z}\right)=\frac{e^{2 x^{z}}-1}{e^{2 x^{z}}+1}
$$

whereas for the output layer was the linear function:

$$
F_{i}\left(s_{j}\right)=s_{j}+w_{j}
$$


The training of the network was carried out using the Back Propagation algorithm. In detail, the learning rates adopted to update the weights of the connections between a layer and the next one were 0.01 , $0.005,0.0025$ proceeding from the input layer to the output one, whereas the momentum factor was always fixed equal to 0.4 .

The data used for modelling the network were collected during 18 consecutive days from $12^{\text {th }}$ to $29^{\text {th }}$ August 2005. During this period the ridge vent was completely open. In detail, data measured from $13^{\text {th }}$ to $28^{\text {th }}$ August were used for training, data of $29^{\text {th }} \mathrm{Au}-$ gust for cross validation and data of $12^{\text {th }}$ August for testing. As the measurement interval was 15 minutes, 96 measures for a day were collected for each parameter. Consequently, among the overall 1728 measures, 1536 were used as training samples, 96 as crossing set and 96 as testing. All the data were normalized in the range $[0,+0.9]$ as the hyperbolic tangent activation function was adopted.

The learning of the network was performed through 10 cycles of training. For each cycle a set of randomized initial values of the weights was used. The maximum number of epochs for each training cycle was 10000 . The ending criterion adopted for the learning process was the cross validation. The number of epochs necessary to stop the training without improvements in the performance of cross validation set was 2000 .

\section{Results and discussion}

\subsection{Reliability of the neural network}

The reliability of the simulation were measured by means of some statistic indicators calculated for each output variable.

In detail, the following indicators were used: the mean, minimum and maximum absolute error, the mean squared error (MSE), the normalized mean squared error (NMSE) and the correlation coefficient (r).

The size of MSE can be used to determine how well the network output fits the desired output, but this measure is not relative, so that the NMSE can be used to compare scores across different data set. The NMSE measure can be regarded as a performance ratio between the simulation obtained with an algorithm (the ANN in this case) and the simple mean of the desired values. The closer NMSE is to 0 the better is the performance of the algorithm. Values above 1 mean that the algorithm is performing even worse than the simple average.

Finally, the correlation coefficient ( $r$ ) is confined to the range $[-1,1]$. When $r$ is equal to 1 there is a perfect positive linear correlation between the measured and the simulated data, which means that they vary by the same amount. When $r$ is equal to -1 there is a perfectly linear negative correlation between the measured and the simulated data, meaning that they vary by the same amount in opposite ways. When $r$ is equal to 0 there is no correlation. Intermediate values describe partial correlations.

Fig. 2 shows the results of the simulations obtained with the neural network compared with the values of the testing set measured on $12^{\text {th }}$ August 2005. In detail, figures $2 \mathrm{a}, 2 \mathrm{c}$ and $2 \mathrm{e}$ report the charts of the $\mathrm{x}$ component values of the air flow speed simulated and measured near the rack, through the ridge vent and through the opening on the paddock respectively. Each chart also represents the $\mathrm{x}$-component of the measured wind values. Similarly, figures $2 b, 2 d$ and $2 \mathrm{f}$ report the corresponding charts for the y-component. Figures $2 \mathrm{~g}$ and $2 \mathrm{~h}$ report the charts of the simulated and measured values of the air temperature and relative humidity inside the dairy house together with the corresponding climatic parameters measured outside.

Table 1 reports for each variable the values of the statistical indicators calculated for the simulation.

The charts and the table show satisfactory results in simulating the air speed components in the considered points inside the building, except for the x-component calculated near the rack.

In detail, the best results in simulating the values of the air speed have been obtained for the ridge vent where the high value of the correlation coefficient, greater than 0.95 for both the components, the mean absolute error not greater than $0.2 \mathrm{~ms}^{-1}$ and the low values of MSE and NMSE indicate a high precision of the simulation. These results are also visible in figures $2 \mathrm{c}$ and $2 \mathrm{~d}$ showing the ability of the network in simulating the curve of the measured air speed even in the presence of sudden variations of its values. Figures $2 \mathrm{c}$ and $2 \mathrm{~d}$ also show the dependence of the air speed through the ridge vent on the wind intensity, that is more evident especially during nighttime.

Less accurate results are provided by the network for simulating the air speed through the opening facing the paddock. In fact, for both the components, the correlation coefficient do not exceed the value of 0.9 , the NMSE values are equal to 0.2773 and 0.2956 for the $\mathrm{x}$ and the $\mathrm{y}$ components respectively, while the mean absolute error is equal to $0.3 \mathrm{~ms}^{-1}$ for both components. Also in this case the results can be checked in figures $2 \mathrm{e}$ and $2 \mathrm{f}$ where the difficulty of the network in simulating the peak values of air speed is noticeable.

The worst result is obtained in the simulation of the $\mathrm{x}$-component of the air speed near the rack. This finding is asserted by a value of NMSE greater than 1 , meaning that the results for this parameter are worthless, and by a correlation coefficient near to 0.60 . Nevertheless, this result has to be interpreted looking at fig. $2 \mathrm{a}$, showing that the $\mathrm{x}$-component values of the air speed are close to zero during all the day. For this reason even very small differences between simulated and measured values lead to high relative errors, although both the mean and the maximum absolute er- 
rors are the lowest than all the other air speed simulations. On the other hand, fig. $2 b$ shows that the $y$ component of the air speed is significantly higher than the $\mathrm{x}$-component. In fact, the openings in the crosswise external walls produce air fluxes generally parallel to the longitudinal axis of the building and often independent from the wind speed and direction. In this case the simulation can be considered satisfactory considering the low value of NMSE close to 0.1 and the correlation coefficient grater than 0.92.

An excellent ability of the network has been obtained in simulating the values of the air temperature and relative humidity inside the dairy house. Fig. $2 \mathrm{~g}$ shows that the air temperature is simulated very well during daytime, while the maximum absolute error, equal to $1.6^{\circ} \mathrm{C}$, occurs during nighttime. Furthermore, simulated and measured data are strongly correlated as shown by the value of the correlation coefficient of about 0.99 . Similarly, fig. $2 \mathrm{~h}$ shows the very good results obtained for the air relative humidity, which simulation gives a correlation coefficient value greater then 0.99 and the lowest NMSE value of the entire simulation.

\subsection{Simulations}

The results of the network testing showed adequate accuracy in the simulation of the air speed across the ridge vent.

As the air-flow rate exchanged across the ridge vent is a crucial issue of the natural ventilation in dairy houses, the neural network was used to investigate how it changes, in relation to the wind speed and direction, performing a parametric analysis.

Taking into account that the training of the network was performed using a set of data referred to a specific period of the year, the parametric analysis was carried out using climatic data reasonably occurring in the summer.

In detail, the wind speed has been varied in the range $0.5 \mathrm{~ms}^{-1} \div 3.5 \mathrm{~ms}^{-1}$ with a step of $0.5 \mathrm{~ms}^{-1}$ and, for each fixed value, the wind direction has been varied between $22.5^{\circ}$ and $337.5^{\circ}$ at intervals of $4.5^{\circ}$. The angular sector of $45^{\circ}$ centred in the $0^{\circ}$ value was excluded from this analysis because the wind never blew from those directions during the trial. Each pair of values was transformed in the chosen Cartesian coordinate system to be provided as input to the network.

The values of outside air temperature and relative humidity were set to $27^{\circ} \mathrm{C}$ and $40 \%$ respectively, corresponding to the rounded mean values of the data measured from 11.00 to 15.00 during the days used for the network training. In this way the simulations were performed under climatic conditions representative of the hottest and sunniest hours of the days considered in the study.

In order to make the illustration of the results easier, the ventilation data obtained from the parametric analysis in the Cartesian coordinate system were transformed in speed and direction values.

Figures $3 \mathrm{a}$ and $3 \mathrm{~b}$ report the parametric curves of air speed and direction through the ridge vent respectively, obtained for each value of wind speed in relation to wind direction.

Figure $3 \mathrm{a}$ shows that the highest speed values of the air flow through the ridge vent are slightly lower than the wind speed and that they occur for wind directions ranging between $76.5^{\circ}$ and $90^{\circ}$. Moreover, from figure $3 \mathrm{~b}$ it results that, in the same range of wind direction, the air flow is always incoming in the dairy house. In fact, the direction of the air flow varies from $87^{\circ}$ to $106^{\circ}$.

For wind directions up to $135^{\circ}$ the simulation keeps on providing high speed values of the air flows incoming in the building. This occurrence confirms that the wind effect is prevalent in comparison to the buoyancy.

For values of wind direction greater than $135^{\circ}$ and up to about $200^{\circ}$, the air speed suffers a rough reduction, aside from the wind speed. Furthermore, in this range, the directions of the air flow and of the wind are fully concordant, so that the air is mainly outgoing from the ridge vent.

At last, for all wind directions greater than $200^{\circ}$, the speed values of the air flow are quite constant and definitely lower than the corresponding speed of the

\begin{tabular}{l|cccccccc}
\hline Performance & $\boldsymbol{T}^{(0)}\left[{ }^{\circ} \boldsymbol{C}\right]$ & $\boldsymbol{R} \boldsymbol{H}^{(0)}[\%]$ & $\boldsymbol{V}_{x}^{(0)}\left[\mathbf{m s}^{-1}\right]$ & $\boldsymbol{V}_{v}^{(0)}\left[\mathbf{m s}^{-1}\right]$ & $\boldsymbol{V}_{x}{ }^{(1)}\left[\mathbf{m s}^{-1}\right]$ & $\boldsymbol{V}_{v}{ }^{(1)}\left[\mathbf{m s}^{-1}\right]$ & $\boldsymbol{V}_{x}{ }^{(2)}\left[\mathbf{m s}^{-1}\right]$ & $\boldsymbol{V}_{v}^{(2)}\left[\mathbf{m s}^{-1}\right]$ \\
\hline MSE & 0.3760 & 8.5640 & 0.0268 & 0.0192 & 0.0698 & 0.1030 & 0.1110 & 0.1524 \\
\hline RMSE & 0.6132 & 2.9264 & 0.1637 & 0.1385 & 0.2642 & 0.3210 & 0.3332 & 0.3904 \\
\hline NMSE & 0.0536 & 0.0280 & 1.6305 & 0.1662 & 0.2131 & 0.1008 & 0.2773 & 0.2956 \\
\hline Mean absolute error & 0.5 & 2.5 & 0.1 & 0.1 & 0.2 & 0.3 & 0.3 & 0.3 \\
\hline Minimum absolute error & 0.0 & 0.0 & 0.0 & 0.0 & 0.0 & 0.0 & 0.0 & 0.0 \\
\hline Maximum absolute error & 1.6 & 10.4 & 0.5 & 0.5 & 0.7 & 0.8 & 0.9 & 0.9 \\
\hline$r$ & 0.9907 & 0.9923 & 0.6070 & 0.9240 & 0.9759 & 0.9519 & 0.9000 & 0.8817 \\
\hline
\end{tabular}

$T=$ air temperature, $R=$ air relative humidity, $V_{x}=x$-component of the air flow speed, $V_{y}=y$-component of the air flow speed

${ }^{(0)}$ by the rack, ${ }^{(1)}$ through the ridge vent, ${ }^{(2)}$ through the opening on the paddock.

TABLE 1 - Statistic indicators to evaluate the performances of the neural network used for the simulations of some microclimatic variables inside the dairy house. 


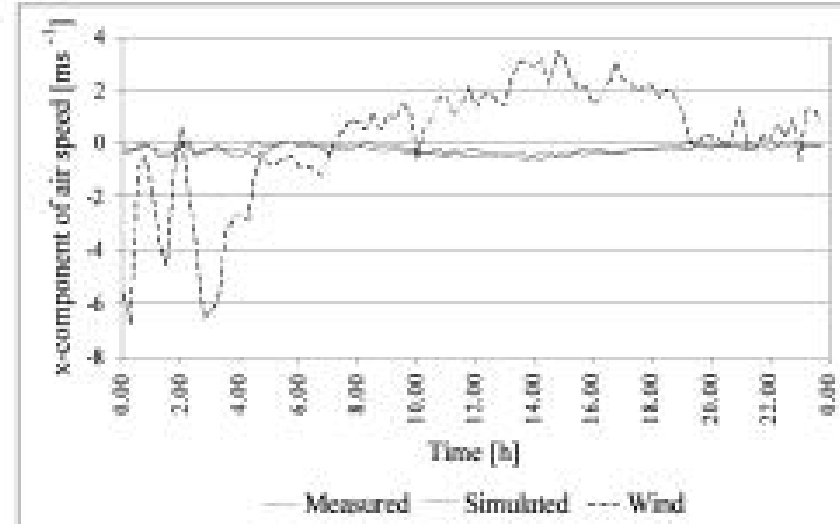

(a)

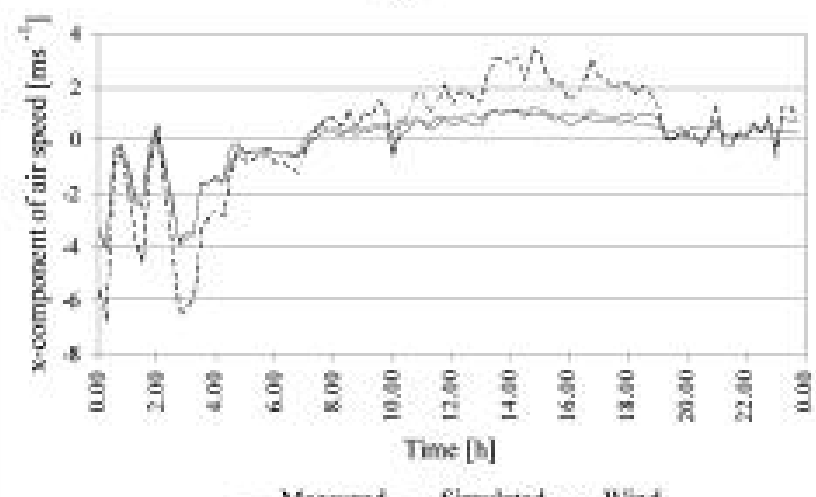

(c)

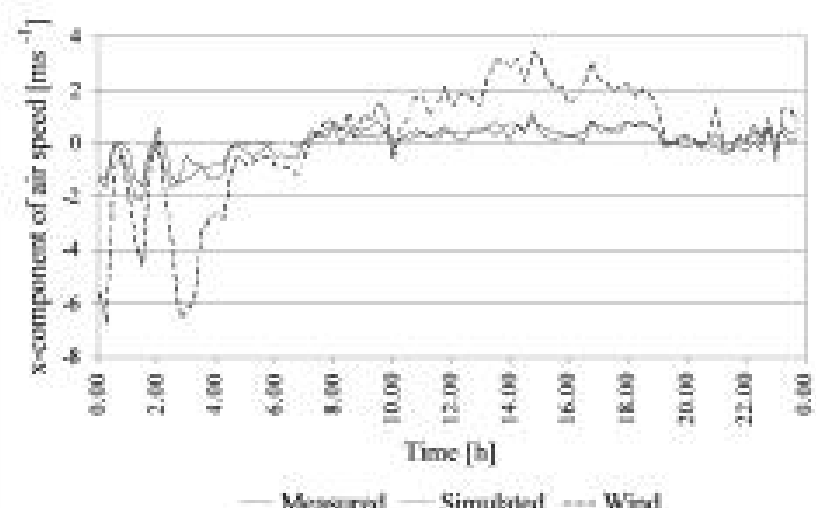

(e)

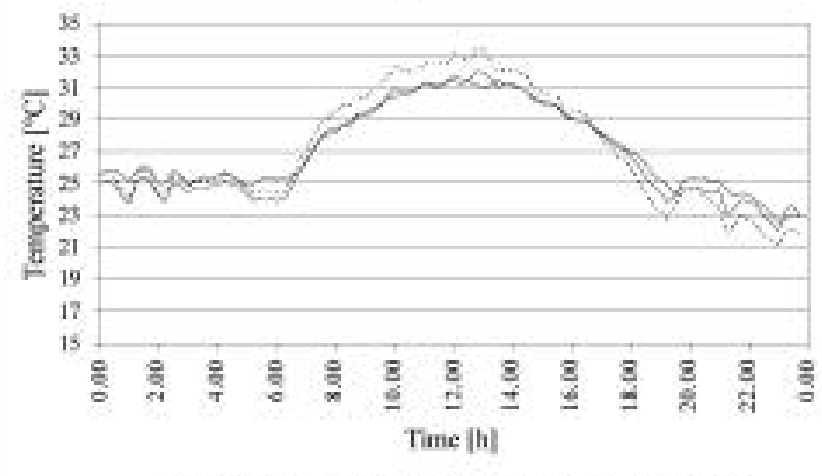

- Measured - Simulaned …. Otside Temperature

(g)

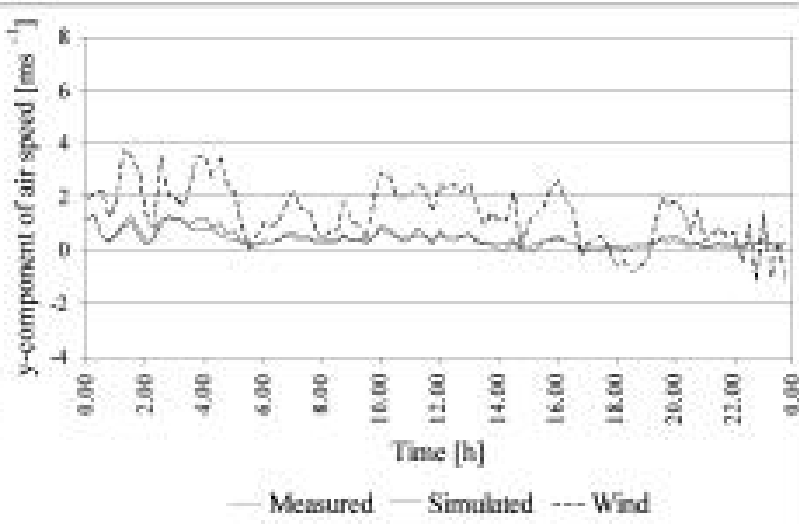

(b)

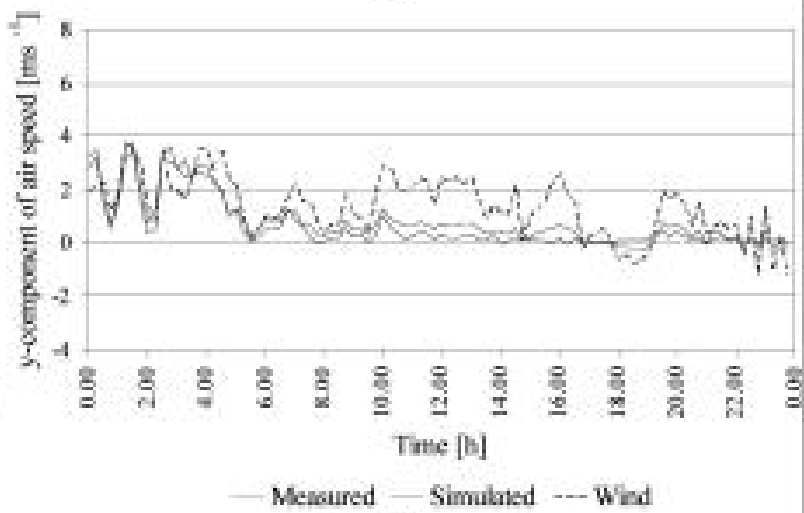

(d)

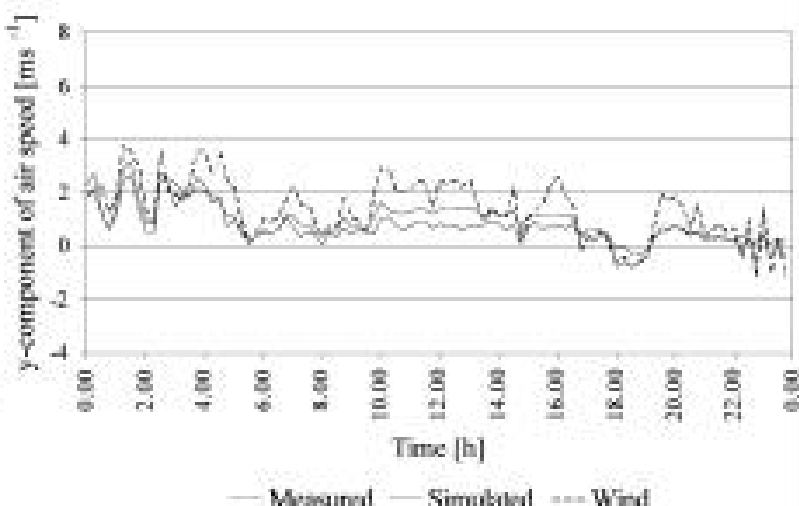

(f)

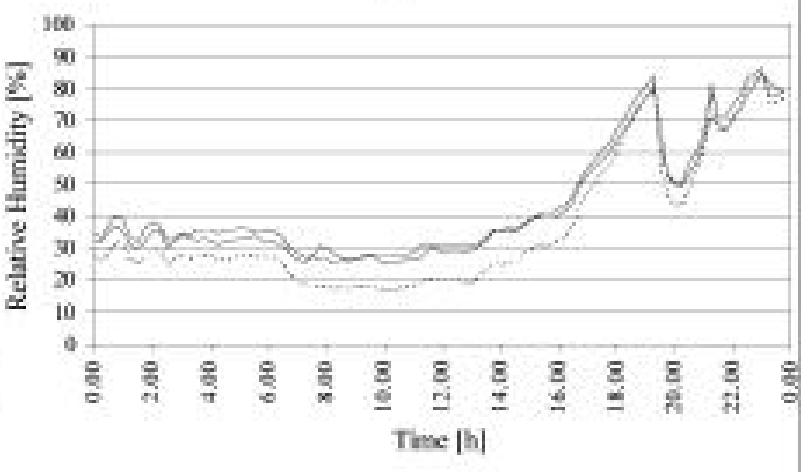

— Measured - Simulated Outside Rebative Humidity

(h)

Fig. $2-X$ and y components of wind speed and air flow speed measured and simulated near the rack (a and $\mathrm{b}$ ), through the ridge vent (c and $\mathrm{d}$ ), through the opened East side (e and $\mathrm{f}$ ), air temperature and relative humidity measured outside the dairy house and the corresponding parameters measured and simulated inside the dairy house ( $\mathrm{g}$ and $\mathrm{h}$ ). 


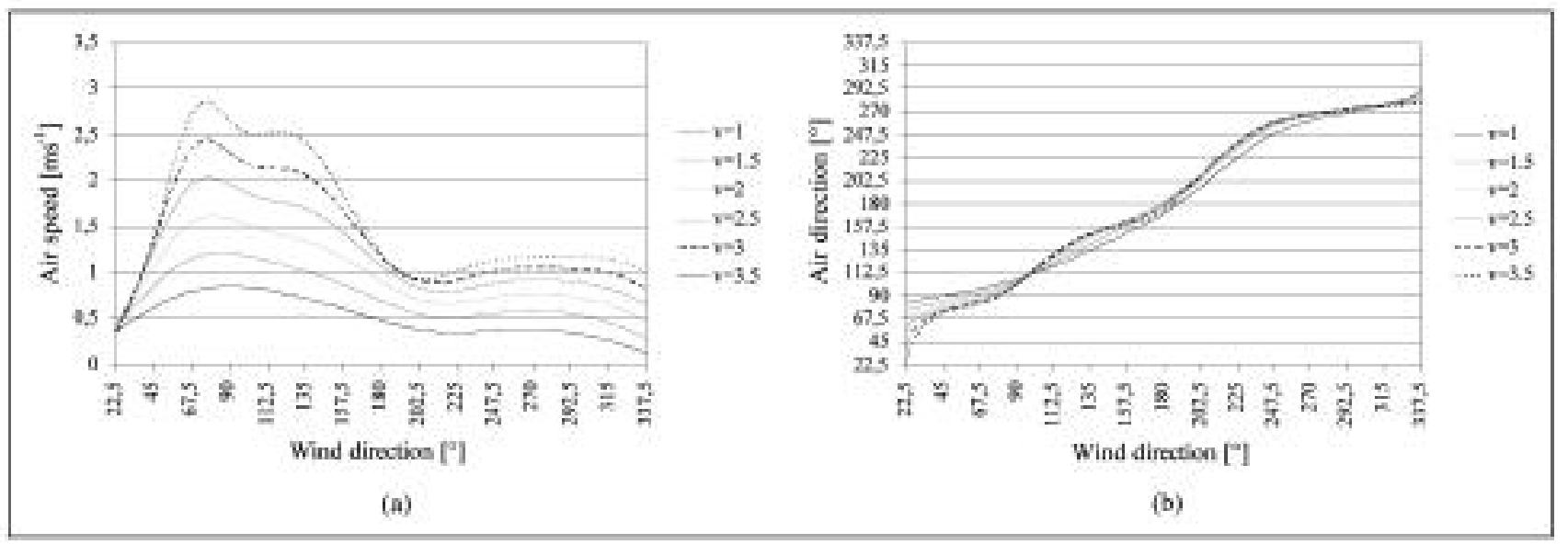

Fig. 3 - Parametric curves of air speed (a) and air direction (b) trough the ridge vent calculated for different values of wind speed in relation to wind direction.

wind. In this range, also the air direction results almost constant and approximately equal to $270^{\circ}$, so indicating the predominance of the chimney effect that causes the leakage of the air perpendicularly to the ridge vent.

\section{Conclusions}

The results of the present study show that the artificial neural networks can be used as an efficient tool to simulate physical and climatic parameters in agricultural buildings. In detail, modelling a Generalized feed-forward Multi-Layer Perceptron ANN allowed to obtain satisfactory results in the simulation of air speed and direction and air temperature and humidity inside a dairy house, using as input the values of wind speed and direction and outdoor air temperature and humidity.

It is reasonable to hold that the architecture of the ANN modelled can be used for simulating physical and climatic parameters inside dairy houses built with materials and geometries different from the ones analyzed in this study and under dissimilar climatic conditions.

The adequate accuracy in the simulation of the air motion across the ridge vent allowed to perform a parametric analysis of the ventilation, which provided the values of air speed and direction in function of a fixed range of values of wind speed and direction.

Forecasting the climatic parameters inside the breeding area can allow to set up and optimize automation systems for controlling ventilation openings inside dairy houses having geometry, building materials and orientation similar to the ones considered in this work.

\section{References}

Bjerg B., Kai P., Morsing S., Takai H., CFD Analysis to Predict Close Range Spreading of Ventilation Air from
Livestock Buildings, Agricultural Engineering International: the CIGR Journal of Scientific Research and Development, 2004, Vol. VI, Manuscript BC 03014.

Bray D.R., Bucklin R.A., Shearer J.K., Montoya R., Giesy R., Reduction of environmental stress in adult and young dairy cattle in hot, humid climates, Proceedings of the Fifth International Symposium Livestock Environmental V, Bloominghton, Minnesota, USA, 29-31 May, 1997, Vol. II, 672-679.

Cammarata S., Reti neuronali. Dal perceptron alle reti caotiche e neuro-fuzzy, Etas Libri, 1997, Milano.

Cascone G., Sistemi passivi ed impianti attivi per il controllo ambientale degli edifici per l'allevamento nei paesi a clima caldo, Medit, 1991, (4), 43-52.

Cascone G., D'Emilio A., Mazzarella R., Criteri di progettazione e gestione elle aperture di ventilazione nelle stalle per bovine da latte mediante modello CFD, Rivista di Ingegneria Agraria, 2006, (1), 25-34.

Cascone G., D’Emilio A., Mazzarella R., Porto S.M.C., Reti neurali per la simulazione di parametri fisici e climatici nelle costruzioni per l'agricoltura, Convegno AIIA 2007 "L'e- nell'ingegneria agraria, forestale e dell'industria agro-alimentare", Firenze, 25-26 Ottobre, 2007.

Frazzi E., Calamari L., Calegari F., Assessment of a thermal comfort index to estimate the reduction of milk production caused by heat stress in dairy cow herds, In Proc. "Fifth international dairy housing conference", Forth Worth, Texas, 29-31 January 2003, 269-276.

Hagivara M., Theoretical derivation of momentum term in back-propagation, Proceedings of the International Joint Conference on "Neural Networks", Baltimore, 7-11 June, 1992, (1), 682-686.

Holmes B.J., Graves R.E.. Natural ventilation for cow comfort and increased profitability, Proceedings of the Third International Dairy Housing Conference, Orlando, Florida, USA, 2-5 February 1994, 397-405.

Kalogirou S.A., Eftekhari M.M., Pinnock D.J., Artificial neural networks for predicting air flow in a naturally ventilated test room, in Building Serv. Eng. Res. Technol., 2001, 22, (2), 83-93.

Kröse B., van der Smagt P., An introduction to neural networks, e-book, 1996, the University of Amsterdam. 
Liberati P., Zappavigna P., Performance of ventilated roofs in hot climate, International Symposium of the CIGR $2^{\text {nd }}$ Technical section, Evora (Portogallo), May 2-6, 2004, 18. CD N. FB04_611.

Rumelhart D.E., Hinton G.E., Williams R.J., Learning representations by backpropagating errors, Nature, 1986 323, 533.

Stowell R.R., Gooch C.A., Bickert W.G., Design parameters for hot-weather ventilation of dairy housing: a critical review, Fifth International Dairy Housing, Texas (USA), January 29-31, 2003, 218-226.

Twomey J.M., Smith A.E., Redfern M.S., A predictive model for slip resistance using artificial neural networks, in IIE Transactions, 1995, (27), 374-381.

Wagenberg A. van, Bjerg B, Bot. G., Measurement and Simulation of Climatic Conditions in the Animal Occupied Zone in a Door Ventilated Room for Piglets, Agricultural Engineering International: the CIGR Journal of Scientific Research and Development, 2004, Vol. VI, Manuscript BC 03020.

Zappavigna P., Liberati P., Slotted roofs as a tool for improving the housing condition in hot climate, Proceedings of the $7^{\text {th }}$ International Symposium "Livestock Environment VII", ASAE, Beijing, China, May 18-20, 2005.1-6. ISBN 1-892769-48-4.

\section{SUMMARY}

The aim of the present paper is to study natural ventilation in a dairy house by means of a parametric analysis relating wind speed and direction to the air flows through the ridge vent of the building.

This analysis was carried out by means of an artificial neural network (ANN) which capability in modelling and simulating some climatic parameters inside a dairy house has been validated using the data collected in a trial carried out during summer 2005.

The results show that modelling a Generalized feed-forward Multi-Layer Perceptron ANN allowed to obtain satisfactory results in the simulation of air speed and direction and air temperature and humidity inside a dairy house, using as input the values of wind speed and direction and outdoor air temperature and humidity.

The adequate accuracy in the simulation of the air motion across the ridge vent allowed to perform a parametric analysis of the ventilation, which provided the values of air speed and direction in function of a fixed range of values of wind speed and direction.

Keywords: natural ventilation, generalized multilayer perceptron, livestock building. 
